\title{
STOCHASTIC MODELING OF RECHARGEABLE BATTERY LIFE IN A PHOTOVOLTAIC POWER SYSTEM
}

\author{
Angel Urbina, Thomas L. Paez \\ Sandia National Laboratories \\ Validation and Uncertainty Quantification Processes, 9133 \\ Mail Stop 0557 \\ Albuquerque, NM 87185-0557 \\ Rudolph G. Jungst \\ Sandia National Laboratories \\ Lithium Battery R\&D Department, 2521 \\ Mail Stop 0613 \\ Albuquerque, NM 87185-0613
}

AIAA-2000-2976

\begin{abstract}
We have developed a stochastic model for the power generated by a photovoltaic (PV) power supply system that includes a rechargeable energy storage device. The ultimate objective of this work is to integrate this photovoltaic generator along with other generation sources to perform power flow calculations to estimate the reliability of different. electricity grid configurations. For this reason, the photovoltaic power supply model must provide robust, efficient realizations of the photovoltaic electricity output under a variety of conditions and at different geographical locations. This has been achieved by use of a Karhunen-Loeve framework to model the solar insolation data. The capacity of the energy storage device, in this case a lead-acid battery, is represented by a deterministic model that uses an artificial neural network to estimate the reduction in capacity that occurs over time. When combined with an appropriate stochastic load model, all three elements yield a stochastic model for the photovoltaic power system. This model has been operated on the Monte Carlo principle in stand-alone mode to infer the probabilistic behavior of the system. In particular, numerical examples are shown to illustrate the use of the model to estimate battery life. By the end of one year of operation, there is a 50\% probability for the test case shown that the battery will be at or below $95 \%$ of initial capacity.
\end{abstract}

\footnotetext{
${ }^{1}$ Copyright $\odot 2000$ by the American Institute of Aeronautics and Astronautics, Inc. All rights reserved.
}

\section{INTRODUCTION}

Renewable energy generators (PV, wind, etc.) are finding increased use for providing power to remote areas that do not have access to the electricity grid. Another useful characteristic of these power sources is low operating cost compared to traditional generation sources such as combustion turbines. Because they can often be sited close to the power demand in distributed generation systems, renewable generators also are claimed to have a positive impact on the reliability of the electricity grid in general. Sandia National Laboratories has been developing power grid models to quantify the reliability of the National power grid and study how it can be improved. In order to include renewable generation in this study, Sandia has developed the stochastic model for a photovoltaic power system.

An energy storage system is necessary to allow the PV generator power to be dispatchable and to continue to service the load during periods of low PV output. The photovoltaic-based power supply must be sized to satisfactorily serve the system load and also to sufficiently charge the energy storage sub-system (typically a lead-acid battery) that will continue to supply the system load while the photovoltaic system is not generating power. Optimal sizing of the system must therefore account for the facts that the power generated by the system and the power used by the load are stochastic processes and that continued deep discharge of the energy storage device can cause accelerated capacity loss in the case of a lead-acid 


\section{DISCLAIMER}

This report was prepared as an account of work sponsored by an agency of the United States Government. Neither the United States Government nor any agency thereof, nor any of their employees, make any warranty, express or implied, or assumes any legal liability or responsibility for the accuracy, completeness, or usefulness of any information, apparatus, product, or process disclosed, or represents that its use would not infringe privately owned rights. Reference herein to any specific commercial product, process, or service by trade name, trademark, manufacturer, or otherwise does not necessarily constitute or imply its endorsement, recommendation, or favoring by the United States Government or any agency thereof. The views and opinions of authors expressed herein do not necessarily state or reflect those of the United States Government or any agency thereof. 


\section{DISCLAIMER}

Portions of this document may be illegible in electronic image products. Images are produced from the best available original document. 
battery. Another use for the stochastic renewable generator model is therefore to provide a predictive capability for the performance of the battery under various design and operating options. This can be used to optimize the system for the best trade off among cost, load requirements, and battery life.

The stochastic photovoltaic power system model will be described for each of its three elements. Then, a numerical example will be presented showing the performance and capabilities of the software. Ultimately the model may be used to design and optimize renewable energy power systems incorporating energy storage.

\section{PV/RECHARGEABLE ENERGY STORAGE MODEL}

The present investigation creates a framework for the analysis of the performance of a photovoltaic power supply system. The power output of the PV array is modeled as a non-Gaussian stochastic process. The stochastic process characteristics are known primarily through statistics of solar insolation taken from data measured at various geographic locations. This investigation models insolation at particular locations using measured statistical data in a Karhunen-Loeve framework. This robust expansion permits the representation of insolation in terms of a small number of deterministic eigenfunctions and non-Gaussian random variable coefficients. Our approach uses a canonical variate analysis (CVA) technique to simulate two main components of the solar radiation, direct normal and diffuse horizontal radiation. In addition, the load profile is modeled as deterministic and/or stochastic, depending on its operating characteristics. The stochastic photovoltaic system and load models allow us to simulate the charge/discharge cycles experienced by the energy storage device during use in the photovoltaic supply environment.

The behavior of the rechargeable energy storage device, in particular the damage caused in a lead-acid battery by prolonged periods of deep discharge, is modeled by means of an artificial neural network (ANN). This deterministic battery model reflects the damage to capacity caused by battery use at low state-of-charge levels. Damage has been observed experimentally, but because the functional form of the relationship between capacity and deep discharge is unknown, and is presumed to vary with battery type, we model it with an ANN whose parameters are to be trained with experimental data. A generalization of the radial basis function ANN is used to represent battery damage as a function of depth of discharge and duration of discharge. Data obtained experimentally serve as exemplars for training the ANN. At this time, only limited battery data that characterize damage associated with discharge periods are available. Therefore, these data were augmented with plausible, synthetic data for training.

All these elements are combined into a single framework to yield a stochastic model for the power supply/energy storage/load system. Figure 1 shows a schematic representation of this system. The model is operated on the Monte Carlo principle to yield realizations of the stochastic processes characteristic of the operational phenomena, and these can be analyzed using the tools of statistics to infer the probabilistic behavior of the system. We shall now discuss the solar resource and energy storage device simulators in more detail.

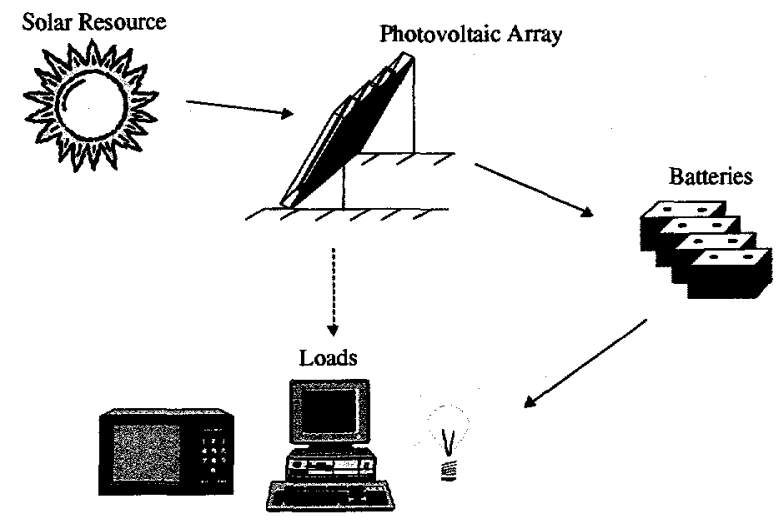

Figure 1: Power supply/energy storage/load system

\section{Solar Resource Simulator}

In previous work, we used a bivariate Markov process to simulate the direct normal and diffuse horizontal solar radiation data. This technique yields accurate realizations of the random process but it is very CPU intensive. In light of this, we seek a faster method that will be as accurate as, or more accurate than our current technique.

The main focus of this investigation is to implement a CVA approach to modeling the daily direct normal and diffuse horizontal radiation components. CVA has been successfully used to model random processes, such as the dynamic response of a nonlinear mechanical system (see Paez and Hunter ${ }^{1}$ ). In the following 
section we describe the CVA approach, and the result of its implementation is presented later in the paper.

\section{Canonical Variate Analysis}

Canonical variate analysis is an extension of the autoregressive moving average (ARMA) modeling technique. Originally developed by Hotelling ${ }^{2}$, CVA was improved by Larimore ${ }^{3}$. Larimore's implementation of CVA permits accurate and efficient simulation of random processes. CVA is described in detail in several references (see Larimore ${ }^{3}$, Hunter ${ }^{4}$ ).

CVA involves three critical transformations, namely, the measurement to state transformation, the evolution of past states to future states, and the future state to estimated measurement transformation. Equations 1 through 4 , below, implement these steps.

Let $\quad \boldsymbol{x}\left(t_{j}\right) j=\ldots,-1,0,1, \ldots$, denote a (possibly multivariate) measured excitation, at times $t_{j}=j \tau, j=\ldots,-1,0,1, \ldots$, and let $y\left(t_{j}\right), j=\ldots,-1,0,1, \ldots$, denote the corresponding (possibly multivariate) measured response. The first operation of CVA transforms measurements of the system "past", $p$, into a state space $s$.

$$
s\left(t_{j}\right)=J p\left(t_{j}\right)
$$

where the system past, relative to time $t_{1}$, is defined:

$$
\begin{aligned}
& p\left(t_{1}\right)=\ldots \\
& {\left[\begin{array}{llllll}
y\left(t_{1}-\tau\right) & \cdots & y\left(t_{1}-l \tau\right) & x\left(t_{1}\right) & \cdots & x\left(t_{1}-j \tau\right)
\end{array}\right]^{T}}
\end{aligned}
$$

The transformation matrix, $J$, will be defined below. The ensemble of past signals, $\boldsymbol{P}$, and the ensemble of future signals, $F$, of the system are defined as follows, relative to time $t_{1}$, in terms of measured excitation and response.

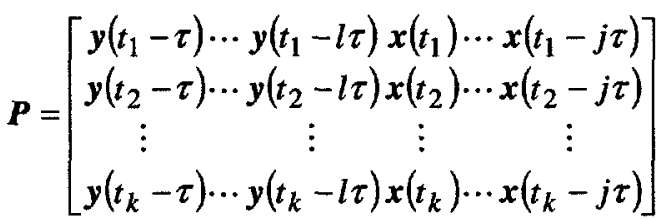

$$
F=\left[\begin{array}{ccc}
y\left(t_{1}\right) & \cdots & y\left(t_{1}+l \tau\right) \\
y\left(t_{2}\right) & \cdots & y\left(t_{2}+l \tau\right) \\
\vdots & & \vdots \\
y\left(t_{k}\right) & \cdots & y\left(t_{k}+l \tau\right)
\end{array}\right]
$$

Past states are evolved into future states, and future states are inverse transformed into the measured response space using the standard modal analysis framework. That framework is characterized by the equations:

$$
\begin{aligned}
s(t+\tau) & =A s(t)+B x(t)+e(t) \\
y(t) & =C s(t)+D x(t)+E e(t)+w(t)
\end{aligned}
$$

where $A, B, C$, and $D$, are system matrices, $e(t)$ and $\boldsymbol{w}(t)$ are noise vectors, and $\boldsymbol{E}$ accounts for state model noise in the state to measurement transformation. The measurement to state transform defined by the matrix $J$ in Equation 1 is developed as follows:

$$
\begin{aligned}
& J=U^{T}\left[\boldsymbol{P}^{T} \boldsymbol{P}\right]^{-1 / 2} \\
& S V D\left[\left[\boldsymbol{P}^{T} \boldsymbol{P}\right]^{-1 / 2}\left[\boldsymbol{P}^{T} \boldsymbol{F}\right]\left[\boldsymbol{F}^{T} \boldsymbol{F}\right]^{-1 / 2}\right]=U W V^{T}
\end{aligned}
$$

where SVD[.] indicates the singular value decomposition. The gist of the operations carried out in these equations is to, first, establish the autocorrelation matrices of the system past, $\boldsymbol{P}$, and future, $\boldsymbol{F}$, using ensembles of segments of the measured excitation and response. These autocorrelations are decomposed using the Karhunen-Loeve expansion (see Ghanem and Spanos ${ }^{5}$ ). The principal components are retained, and their cross-correlations are orthogonalized. This yields the transformation matrix, $J$, a measurement to state space transformation that yields an optimal relation between past and future principal components. Next all measured data segments are transformed into the state space using $J$. The state transition and other state space matrices, $A, B, C$, and $D$, are identified using linear least squares. The transformation and state space parameters are identified using $\boldsymbol{P}$ and $\boldsymbol{F}$, ensembles of signals. Response predictions operate on $p$ to predict $f$.

A global model can be created using all the data, or a local model can be created using data in the neighborhood of a particular past, $p$. In this way, we create global and local linear models. In this investigation, local linear models are used to characterize the joint behavior of direct normal and diffuse horizontal insolation. Further, we take the excitation, $y$, to be zero in this application. 
Equation 4 results from minimizing the mean square error in the prediction of the future $f$ from the past $p$. The process of transforming the measurements $p$ to critical waveforms $s$, transforming past states $s$ to future states, and finally returning to the measurement domain for $f$ may seem awkward, but in fact is considerably more stable than directly predicting $f$ from $p$. Intuitively, the gain comes from minimizing the number of parameters necessary in the past to future prediction process.

With the approach outlined above, we generate realizations of daily direct normal and diffuse horizontal radiation and subsequently use them as the power source for our model. In the next section we examine the energy storage component of our model.

\section{RECHARGEABLE ENERGY STORAGE MODEL}

A lead-acid battery will serve as the energy storage device for this model, allowing the power from the PV source to be dispatchable. Due to the random nature of the PV resource, it is expected that the battery will experience periods of deficit charging, which are known to cause a degradation of the battery capacity. If these periods are frequent, they can eventually lead to battery failure. One goal of this research is to model this damage mechanism via a non-phenomenological technique, in this case using an ANN. The following sections describe the particular ANN used in this work.

\section{Artificial Neural Networks}

A focus of this investigation is to use an ANN to model the damage caused in a rechargeable lead-acid battery due to extended discharge periods. Due to the complex behavior of a rechargeable battery, an explicit form relating the capacity loss to the depth and duration of a discharge cannot be easily derived. This is partly due to the expected variability in the results obtained from the different batteries that will be tested and partly due to the complexity of the electrochemical reactions involved in the process. It is thus convenient to simulate the experimental data using a nonphenomenological modeling technique to characterize the relationship between the inputs and desired outputs. In this case, the output is the damage introduced to the battery due to discharge cycles and the inputs are the duration and depth of the discharge. Previous work, such as O'Gorman et $a l^{6}{ }^{6}$, Urbina et $a l^{7}$, Chan et $a l^{8}$, Yamazaki et $a l^{9}$ and Peng et $a l^{10}$, have successfully demonstrated the modeling of primary and secondary battery systems using ANN. In the following section, a brief presentation of the specific ANN algorithm used in this project is given.

\section{The Multivariate Polynomial Spline ANN}

The multivariate polynomial spline (MVPS) network is an artificial neural network of the radial basis function type. The radial basis function ANN, which was developed by Moody and Darken ${ }^{11}$, simulates mappings via the superposition of radial basis functions. The radial basis function ANN is an accurate local approximator, and although it trains rapidly, it has the potential for size difficulties as the dimension of the input space grows. A generalization of the radial basis function ANN is the connectionist normalized linear spline (CNLS) network. This was developed by Jones et al. ${ }^{12}$ and seeks to simulate a mapping by using radial basis functions in a higher order approximation than the radial basis function network. The MVPS network generalizes the CNLS network to multiple output dimensions and higher degree local approximations. The development of the mathematical framework behind the MVPS network is presented in the following.

First, let $X$ be an n-dimensional input vector to the system being modeled, and let $Z$ be its corresponding m-dimensional output vector. The mapping from $X$ to $Z$ is denoted by:

$$
Z=g(X)
$$

Assume that the function $g(X)$ is deterministic but that its form and parameters are unknown. Write an identity that equates $g(X)$ to itself, then multiply both sides by a weighting function (radial basis function) that is centered at $C_{j}$ :

$$
\begin{aligned}
& g(\boldsymbol{X}) w\left(\boldsymbol{X}, \boldsymbol{C}_{j}, \boldsymbol{\beta}\right)=g(\boldsymbol{X}) w\left(\boldsymbol{X}, \boldsymbol{C}_{j}, \boldsymbol{\beta}\right) \\
& j=1, \ldots, N
\end{aligned}
$$

Expand the function $g(X)$ on the right hand side of Equation 6 using the first three terms in the Taylor series:

$$
\begin{aligned}
& g(\boldsymbol{X}) w\left(\boldsymbol{X}, \boldsymbol{C}_{j}, \beta\right) \cong \\
& {\left[\boldsymbol{A}_{0 j}+\boldsymbol{A}_{1 j}\left(\boldsymbol{X}-\boldsymbol{C}_{j}\right)+\boldsymbol{A}_{2 j} \zeta\right] w\left(X, C_{j}, \beta\right)}
\end{aligned}
$$

where $A_{0}, A_{1}$ and $A_{2}$ are the coefficients of the local models, $\boldsymbol{C}_{j}$ is the "center" of the $\boldsymbol{j}^{\text {th }}$ local approximation (also referred to as center vector or center), $\zeta$ contains 
the quadratic and cross terms obtained from the elements in $X, \beta$ is a parameter related to the width of the radial basis function and $w\left(X, \mathcal{C}_{\mathrm{j}}, \beta\right)$ are the weights attached to the local models (in this case a multivariate Gaussian probability density function is chosen as the weighting expression).

This approximation can be optimized in the neighborhood of $\boldsymbol{C}_{\boldsymbol{j}}$ using least squares or weighted least squares and is accurate in the vicinity of the data used to develop it as long as the behavior of the mapping in the neighborhood is closely represented by the Taylor series. Approximations can be developed in all neighborhoods of the input vector space. The subscript $j$ (where $j=0,1 \ldots N$, and $N$ is the number of regions of approximation) accounts for this in Equations 6 and 7.

The local approximations of the $X$ to $Z$ mapping can now be combined to create an approximate, global map. To accomplish this, several of the approximations are superimposed in a series. Each component in the series is weighted according to its distance from the input vector $\boldsymbol{X}$. Local models that are near $\boldsymbol{X}$ are weighted heavily, whereas those that are further away are weighted less. The series is obtained by summing the terms in Equation 3 as follows:

$$
\begin{aligned}
& \sum_{j} g(X) w\left(X, C_{j}, \beta\right) \cong \\
& \sum_{j}\left[A_{0 j}+A_{1 j}\left(X-C_{j}\right)+A_{2 j} \zeta\right] w\left(X, C_{j}, \beta\right)
\end{aligned}
$$

Since $g(X)$ on the left-hand side of Equation 8 is independent of the index $j$, it can be removed from the summation. The resulting expression can be normalized to obtain:

$$
g(X) \cong \frac{\sum_{j}\left[A_{0 j}+A_{1 j}\left(X-C_{j}\right)+A_{2 j} \zeta\right] w\left(X, C_{j}, \beta\right)}{\sum_{j} w\left(X, C_{j}, \beta\right)}
$$

Equation 9 represents the parametric form of the MVPS network. Figure 2 shows a single input/single output example of local polynomial models fitted to arbitrary input/output data. It can be seen from the figure that for a highly nonlinear response, the local polynomial modeling is quite appropriate. Ultimately, and as described in the above paragraphs, the local models are splined together to form a global model.
The MVPS network is used in feed forward operation by specifying the input vector $\boldsymbol{X}$, evaluating the weights $w$, calculating $\zeta$, substituting the weights and $\zeta$ into Equation 9, and evaluating the output $g(X)$. This output is an interpolation among the training outputs. Note that the range of the summation index $j$ is not specified in Equation 9. It is clear that the summation should be carried out over those local models nearest the input vector $\boldsymbol{X}$. The ANN computer code used in the present investigation accepts a user-defined number of local models and the ones nearest, in Cartesian space, to the input vector are chosen to make each prediction. Using the MVPS ANN, we developed a model to represent the damage that accumulates in a lead-acid battery when exposed to periods of deficit charging.

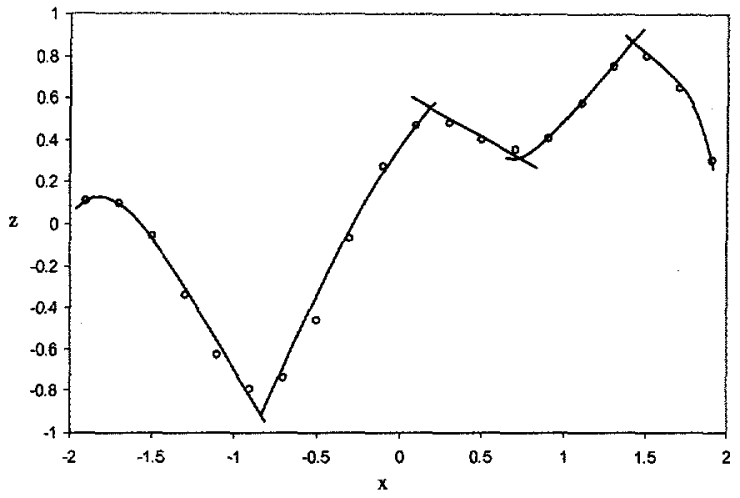

Figure 2: Local polynomial models

\section{ANN-Based Lead-Acid Battery Damage Model}

The objective of the rechargeable battery portion of the power supply model is to simulate the loss of capacity of the battery with time as it is subjected to a cycling environment typical of photovoltaic applications. In addition to the normal wear out of the battery that occurs as a function of number of cycles, depth of discharge, discharge rate, and temperature, batteries are frequently exposed to periods of deficit charging in PV applications. These accelerate degradation of capacity in lead-acid systems due to sulfation of the battery plates. It is assumed in this study that deficit charging is the major factor causing accelerated loss of capacity and therefore shortened life of batteries in PV power supplies. Experimental measurements will be aimed at determining what particular deficit charge conditions have the greatest effect on capacity loss. Two parameters were selected to characterize the deficit charge, namely maximum depth of discharge, $D$, and the duration of the discharge, T. Figure 3 shows these variables. 
The maximum depth of discharge, $\mathrm{D}$ is defined as the deepest discharge, (as measured from a reference threshold, $C_{d}$ ) encountered during a deficit charge period. The duration of the discharge, $T$ is defined as the time it takes to return the capacity to the reference level, $C_{d}$. (This is a function of the amount of solar insolation available.) A moving average was used to represent the average daily capacity at any time.

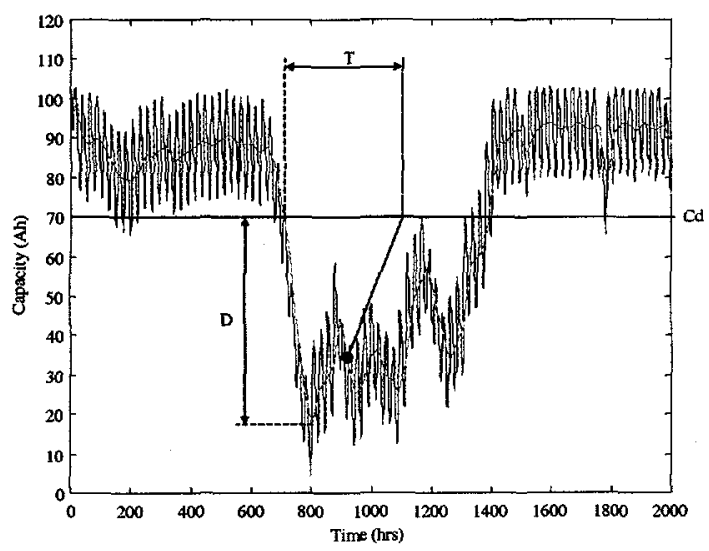

Figure 3: Definition of depth and duration of discharge

For initial calibration of the battery damage, the model draws on data collected earlier at the Florida Solar Energy Center (FSEC). Different charge to load ratios and discharge rates were studied to determine the effect on cycle life. For details on these tests see Reference 13. Although these data give some indication of the reduction in cycle life that occurs due to extended times at low state-of-charge (SOC), there are too few data points to completely define the battery damage surface. Consequently, a new set of deficit charge cycle tests has been planned that will provide a large enough data set to complete training of the ANN.

The focus in the new tests is to be on the damage due to deficit charging, so only a single discharge rate, $\mathrm{C} / 20$, will be used. A few sustaining cycles will be run before and after the deficit charge region before measuring the capacity loss; however, batteries will not be cycled to end-of-life. This will shorten the experiments to a few weeks maximum and will allow a greater variety of deficit charge characteristics (depth and duration of discharge) to be examined. Complex impedance data will also be collected to investigate whether small amounts of battery degradation can be detected earlier in the tests by this method.

Figure 4 shows the battery damage model which we simulated using an MVPS ANN. As experimental measurements of battery capacity loss are completed, the ANN description of the damage surface can be easily updated and refined. It is important to note that although reasonable, this initial model is only based on limited data and must be revised.

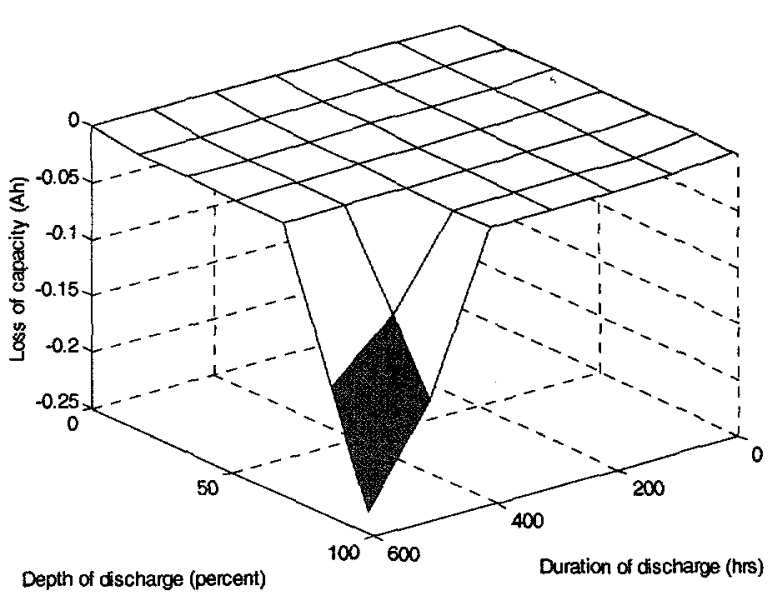

Figure 4: Proposed battery damage model

\section{TEST CASE AND RESULTS}

A test case was set up to exercise the PV/rechargeable battery/load model. The model was operated on the Monte Carlo principle to yield realizations of the hourly solar insolation data, using the CVA technique described earlier. The solar insolation along with the load profile was input to the lead-acid battery model and hourly capacity cycling data were produced.

The specific test case consisted of a PV array located in Albuquerque, NM (latitude $35^{\circ} 03^{\prime}$ ). The tilt angle was set at $50^{\circ}$ (which enhances power generation in the winter months) and the azimuth angle was set to $0^{\circ}$ (which means that the array is facing due South). The PV array is rated at 2.1 amps at standard test conditions (i.e. $1000 \mathrm{w} / \mathrm{m}^{2}$ and $25^{\circ} \mathrm{C}$ temperature) and has a nominal voltage of 12 volts. Three PV modules were connected in parallel to give a current of 6.3 amps. A 105 Ah lead-acid battery serves as the energy storage device for this example. It is assumed that the capacity threshold, $\mathrm{C}_{\mathrm{d}}$, below which a discharge starts to cause damage, is $52 \mathrm{Ah}$ ( $50 \%$ of initial capacity). The battery damage surface shown in Figure 4 is simulated via the MVPS ANN. The applied load consists of a constant load during nighttime and a randomly applied load during the daytime. This load profile requires an average of $30 \mathrm{Ah}$ per day/night. 
The following results were obtained using simulated PV data based on 10 years of actual insolation measurements for the Albuquerque, NM area.

Figure 5a shows measured hourly insolation data for the month of January 1978. Figure 5b shows a realization of hourly insolation data for a simulated January as obtained with our PV simulator software. It is important to note that these realizations will not be identical because they are random process realizations, but their characters should be similar because the random process source of Figure $5 \mathrm{~b}$ is meant to simulate the random process source of Figure 5a. That is, there are days in which the insolation is low and others in which it is high.

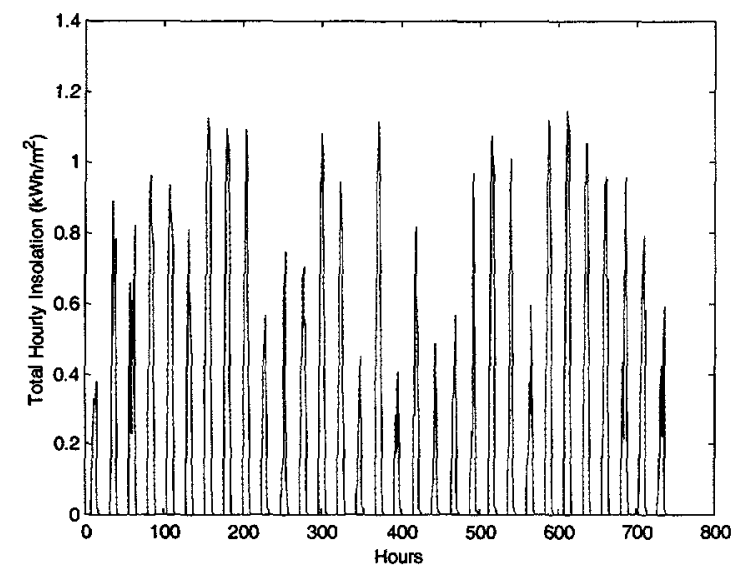

Figure 5a: Measured total hourly insolation for January 1978

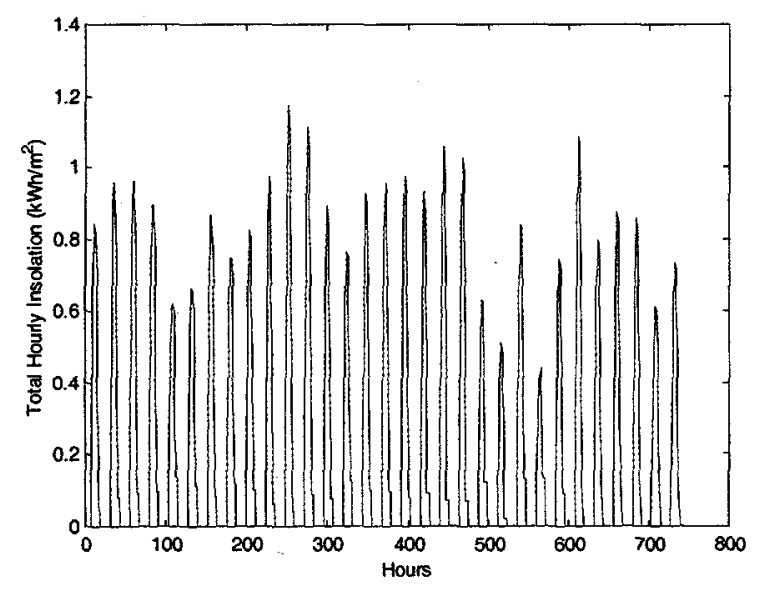

Figure 5b: Simulated total hourly insolation for 31 January days

Figure 6 shows a time history for one simulated month of estimated available capacity data (shown as a solid line) and the maximum potential capacity, $M$ (shown as a heavy solid line). $M$ is the initial capacity minus the irreversible capacity loss that accumulates due to deep discharge events and also due to regular daily cycling. As expected, there is a drop in the maximum potential capacity where the deep discharges occur. This is consistent with our ANN model shown in Figure 4. That is, more damage is caused to the battery during deep discharges with long duration.

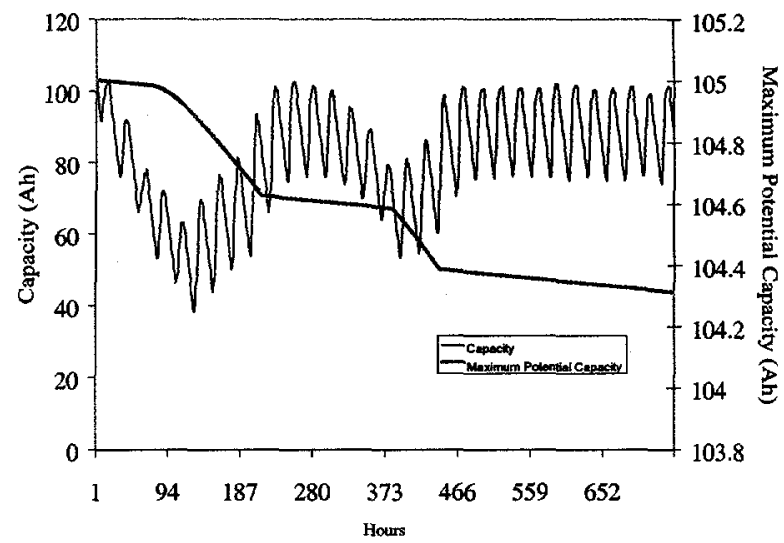

Figure 6: Capacity simulation using a random load

A first passage probability analysis was performed for the maximum potential capacity, $M$, and it is shown in Figure 7 . This shows the probability that a battery passes a specific capacity threshold prior to any given time. The results show that by approximately 4500 hours of operation (approximately 6 months), the battery's maximum potential capacity will be below $99 \%$ of the initial capacity with probability one. By the end of one year of operation, there is about an $80 \%$ probability that the battery will be at or below $97 \%$ of the initial capacity and about $50 \%$ probability that it will be at $95 \%$ or less of the initial capacity.

Figure 7 also shows a comparison of first passage probability distributions for maximum potential capacity, M, at several different barrier levels. The solid lines are the first passage probability distributions that result from Monte Carlo analysis of 100 insolation realizations. The dashed lines are the first passage probability distributions that result from Monte Carlo analysis of ten years of measured insolation data. (The latter curves possess a stepped character because the number of years of data measurement is relatively small.) Some possible reasons for disagreement in the short term are (1) the limited amount of measured data, and (2) the detailed behavior of the simulation. Importantly, as the probability of first passage failure increases, the results of the simulations converge to the results based on measured data. 
This type of analysis allows us to make decisions such as when the optimal time to replace a battery has been reached and how to size the PV/battery system to achieve the desired reliability for a particular application.

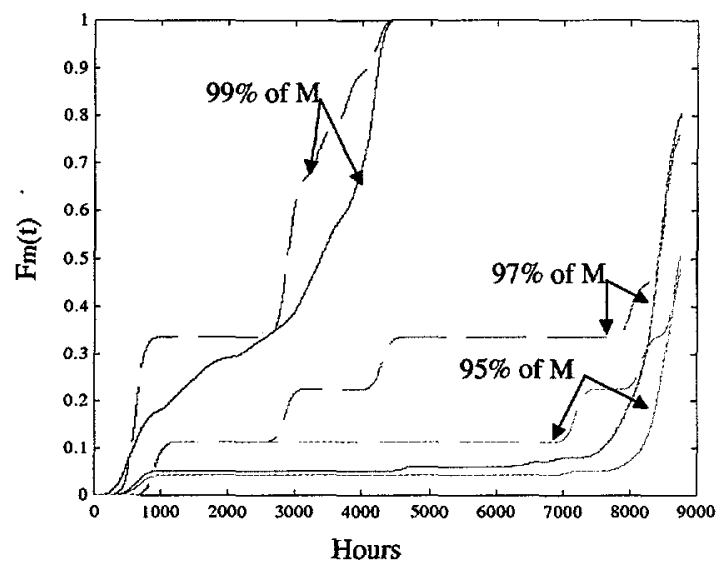

Figure 7: First passage probability of $\mathrm{M}$ (solid line = using simulated data; dashed line = using actual data)

One of our objectives in implementing the CVA technique was to reduce the computational time required to generate yearly realizations of the solar insolation. Using the Markov approach of reference 7, the CPU time to generate a year-long realization was 2.4 minutes using a. Using this new CVA approach the time is reduced to 1.2 minutes without a loss in accuracy. Both examples were run on a HP Vectra PC running at $400 \mathrm{MHz}$. This time reduction is critical since our PV generator will eventually be integrated into a larger power flow analysis code.

\section{SUMMARY}

The objective of this investigation was to develop a framework for simulating the power output from a photovoltaic system, including an energy storage device. The model has also been demonstrated to be capable of assessing the reliability and life of rechargeable batteries in the $\mathrm{PV}$ use environment. This information can be applied to optimize PV system designs and establish more cost-effective battery replacement schedules. A means for simulating the solar insolation random process was required, and we sought an efficient technique for the generation of random process realizations, where the random process is only characterized by historical records of the phenomenon. We chose to use the canonical variate analysis approach, a technique that has been widely used to simulate dynamical systems. The CVA approach was used to generate realizations of the two main components of solar insolation.

The current system uses lead-acid batteries for energy storage. It is acknowledged that damage can accumulate in rechargeable batteries when they are used in a deficit charge environment, such as PV. Damage modeling was performed using a multivariate polynomial spline artificial neural network. The system load was modeled as a combined deterministic/stationary random process.

The results of the investigation and numerical examples indicate that plausible simulations of available battery capacity and maximum potential capacity, $M$, can be generated. Indeed, the important measure of model performance embodied in the first passage probability distributions for $M$ shows that the system performance simulations are accurate. Calculations for a test case predict good retention of battery capacity for at least one year. It remains to investigate more metrics of system performance to assure that system simulations are accurate in all details.

\section{ACKNOWLEDGEMENTS}

Sandia National Laboratories is a multi-program laboratory operated by Sandia Corporation, a Lockheed Martin Company, for the United States Department of Energy under Contract DE-AC04-94AL85000.

\section{REFERENCES}

1. Paez, T., Hunter, N., (1999), "Dynamical System Modeling Via Signal Reduction and Neural Network Modeling," Proceedings of the $70^{\text {th }}$ Shock and Vibration Symposium.

2. Hotelling, H., (1936), "Relations Between Two Sets of Variates," Biometrica, V. 28, pp. 321-377.

3. Larimore, W., (1983), "System Identification, reduced Order Filtering, and Modeling Via Canonical Variate Analysis," Proceedings of the American Control Conference, H. Rao, P. Dorato, eds.

4. Hunter, N., (1997), "State Analysis of Nonlinear Systems Using Local Canonical Variate Analysis," Proceedings of the $30^{\text {th }}$ Hawaii International Conference on System Science. 
5. Ghanem, R., Spanos, P., (1991), Stochastic Finite Elements: A Spectral Approach, Springer-Verlag, New York.

6. O'Gorman, C. C., R. G. Jungst, D. Ingersoll, and T. L. Paez, (1998), "Artificial Neural Network Simulation of Battery Performance", Proceedings of the $194^{\text {th }}$ Electrochemical Society Meeting, Abstract \#66.

7. Urbina, A., T. L. Paez, C. C. O'Gorman P. Barney, R. G. Jungst and D. Ingersoll, (1999), "Reliability of Rechargeable Batteries in a Photovoltaic Power Supply System", Journal of Power Sources 80 , pp. 30-38.

8. Chan, C. C., E. W. C. Lo, and S. Weixiang, (2000), "The available capacity computation model based on artificial neural network for lead-acid batteries in electric vehicles," Journal of Power Sources 87, pp. 201-204.

9. Yamazaki, T., K. Sakurai, and K.-I. Muramoto, (1998), "Estimation of Residual Capacity of sealed Lead-Acid Batteries by Neural Network," Proceedings of the Twentieth International Telecommunications Energy Conference, pp. 210214.

10. Peng, J., Y. Chen, and R. Eberhart, (2000), "Battery Pack State of Charge Estimator Design Using Computational Intelligence Approaches," Proceedings of the Fifteenth Annual Battery Conference on Applications and Advances, pp. 173-177.

11. Moody, J. and C. Darken, (1989), Neural Computation, (1) 2, pp. 281-294.

12. Jones, R. D., et al., (1990), Cognitive Modeling in System Control, The Santa Fe Institute.

13. Hund, T., (1996), Battery Testing for Photovoltaic Applications, Sandia National Laboratories, report number: SAND96-2794C. (This report can also be found at the following Internet address: http://www.sandia.gov/pv/bos/batteries.htm). 